\title{
Comparison of Candida ID medium with Sabouraud-chloramphenicol agar for the isolation of yeasts from clinical haematology surveillance specimens
}

\author{
W. J. OLVER, J. STAFFORD, P. CHEETHAM and T. C. BOSWELL \\ Department of Microbiology and Public Health Laboratory, City Hospital, Hucknall Road, Nottingham NG5 1PB
}

\begin{abstract}
Candida ID is a new chromogenic medium for the identification of yeasts from clinical specimens. $C$. albicans produces blue pigmentation, whereas pink pigmentation is produced by $C$. tropicalis, $C$. Iusitaniae, $C$. guilliermondii and $C$. kefyr; other Candida species appear white. In this study, 240 clinical samples (throat swabs and stool samples) from haematology patients were inoculated on to Candida ID and Sabouraudchloramphenicol agar in parallel, yielding a total of 105 yeasts; the media had overall detection rates of $85.7 \%$ and $86.7 \%$ respectively. The sensitivity of Candida ID for identification of $C$. albicans by blue pigmentation was $52.9 \%$ at $24 \mathrm{~h}$ and $94.1 \%$ at $48 \mathrm{~h}$. Specificity of the blue pigmentation was $100 \%$ at $48 \mathrm{~h}$. Two strains of $C$. tropicalis were identified, one produced pink pigmentation at $72 \mathrm{~h}$, the other strain did not produce any pigmentation after 5 days. Candida ID was superior in detecting mixtures of yeasts compared with Sabouraud-chloramphenicol agar. Candida ID is a suitable primary isolation medium for yeasts from clinical specimens, providing rapid direct identification of $C$. albicans and enhanced detection of mixtures.
\end{abstract}

\section{Introduction}

Infections caused by yeasts and moulds are increasing because of the rising number of immunocompromised patients. Candida species are now the fourth commonest cause of bloodstream infection in the USA [1]. Although C. albicans is still the commonest cause of candidaemia, some studies have found increasing numbers of infections with non-albicans species [13], e.g., C. krusei and C. glabrata, which can be highly resistant to fluconazole. Most clinical laboratories use the germ-tube test as the initial test to differentiate $C$. albicans from other yeasts, followed by time-consuming and expensive biochemical identification, with full identification taking up to $72 \mathrm{~h}$ from primary isolation of the organism. Therefore, there is a requirement for the prompt identification of Candida species in clinical specimens.

Chromogenic media allow rapid presumptive identification of yeasts on primary plates. Several formulations

Corresponding author: Dr W. J. Olver (e-mail: mrzwo@ gwmail.nottingham.ac.uk).

Received 29 May 2001; revised version accepted 4 Sept. 2001. have been evaluated. Albicans ID (bioMérieux, UK) contains a chromogenic substrate for the rapid identification of $C$. albicans. Colonies appear blue, and sensitivity and specificity have both been found to be $>90 \%[4,5]$ from clinical specimens. CHROMagar Candida (CHROMagar S.A., France) allows identification of $C$. albicans (green colonies), as well as $C$. tropicalis (blue colonies) and C. krusei (pink colonies). Sensitivity and specificity have been found to exceed $99 \%$ for all three species [6]. Also, the detection of mixtures of yeasts with both media is superior to Sabouraud-chloramphenicol agar [5,7]. Candida ID is a recently developed medium (bioMérieux, UK). In addition to producing blue coloration of $C$. albicans (having the same chromogenic substrate as Albicans ID), it contains a second substrate, hydrolysis of which yields pink colonies. Colonies of $C$. tropicalis, $C$. lusitaniae, C. guilliermondii and C. kefyr are pink after incubation for $48 \mathrm{~h}$; other yeast species are white. Evaluation of Candida ID showed that it allowed the isolation of more Candida species than Albicans ID, with $97.6 \%$ sensitivity and $89.7 \%$ specificity in the detection and differentiation of $C$. albicans [8]. The aim of the present study was to perform an independent evaluation of Candida ID compared with Sabouraudchloramphenicol agar for culture of surveillance speci- 
mens from patients at high risk of invasive yeast infection. Throat swabs and stool samples were taken from patients with haematological malignancies and bone marrow transplantation. The medium was also evaluated with simulated mixed cultures.

\section{Materials and methods}

\section{Recovery rates of yeasts on chromogenic medium}

Four clinical isolates (C. albicans, C. glabrata, $C$. tropicalis and Saccharomyces cerevisiae) were each suspended in $5 \mathrm{ml}$ of nutrient broth at $c .10^{5} \mathrm{cfu} / \mathrm{ml}$ concentration (turbidity just visible). A 1 in 100 dilution was made and $20 \mu \mathrm{l}$ of this suspension were inoculated on to a quarter plate of blood agar, Sabouraud-chloramphenicol agar and Candida ID for each strain (estimated 20 colonies). The experiment was performed in triplicate. Plates were incubated at $37^{\circ} \mathrm{C}$ in air for $48 \mathrm{~h}$ and colonies were counted.

\section{Ability of chromogenic medium to distinguish mixed cultures of yeasts}

The reliability of distinguishing blue, white and pink colonies on Candida ID was tested by simulation of a mixed clinical sample. Varying concentrations of yeasts, with strains of $C$. albicans (blue), C. glabrata (white) and $C$. tropicalis (pink) were added to portions of a stool sample known to be culture-negative for yeasts, in different combinations. Equal mixtures of organisms were used, as well as combinations where one strain was at a 10-fold lower concentration than another. Stool samples were plated on to Candida ID and incubated for $72 \mathrm{~h}$.

\section{Detection and presumptive identification of yeasts from clinical specimens}

A total of 240 clinical samples (150 throat swabs, 90 stool samples) was collected from 68 inpatients on the haematology unit at Nottingham City Hospital over a period of 3 months. Samples were taken and sent to the laboratory on a weekly basis. Throat swabs were processed by breaking off the end of the swab into $1 \mathrm{ml}$ of nutrient broth. The sample was then agitated on a vortex mixer for $15 \mathrm{~s}$. One drop of suspension was inoculated on to one half of Candida ID and Sabouraud-chloramphenicol agar plates. Stool samples were processed by emulsifying a pea-sized portion of the sample in $5 \mathrm{ml}$ of Ringer's solution. One drop of suspension was inoculated on to one half of Candida ID and Sabouraud-chloramphenicol agar plates. Plates were spread for discrete colonies and incubated at $37^{\circ} \mathrm{C}$ in air. Plates were read independently, by different individuals, after incubation for 24 and $48 \mathrm{~h}$ and again on day 5. Mixed cultures were identified by observation of differences in colony size, texture or colour. Colonies were identified by the germ-tube test with horse serum and read after incubation for $2-4 \mathrm{~h}$ at $37^{\circ} \mathrm{C}$ in air. Germ-tube-negative isolates were then identified by the API 20C AUX system (bioMérieux).

\section{Statistical methods}

Direct identification of $C$. albicans on the two media was analysed in terms of sensitivity - number of true positives/(number of true positives + number of false negatives) - and specificity - number of true negatives/(number of true negatives + number of false positives). Detection rates on the two media were compared by the standard error of difference between percentages.

\section{Results}

\section{Recovery rates of yeasts on chromogenic medium}

Candida ID was not significantly different from Sabouraud-chloramphenicol in the overall recovery of yeasts (Table 1). However, it was noted that colonies of $S$. cerevisiae were smaller on Candida ID medium (c. $\leqslant 1 \mathrm{~mm}$ ) than on Sabouraud-chloramphenicol.

\section{Ability of chromogenic medium to distinguish mixed cultures of yeasts}

The ability to distinguish between blue, white and pink colonies on Candida ID from simulated mixtures of yeasts in clinical samples was good. After incubation for $24 \mathrm{~h}$, colonies of $C$. tropicalis were faint pink in colour and difficult to distinguish from colonies of $C$. glabrata (white). However, after $48 \mathrm{~h}$, pink colonies were easily identifiable, even when mixed with other yeasts in a 1:10 ratio. Blue (C. albicans) and white colonies were easily distinguished after incubation for $24 \mathrm{~h}$. In mixtures of all three yeasts, blue and white colonies were recognisable after $24 \mathrm{~h}$, with pink colonies identified after $48 \mathrm{~h}$.

Table 1. Yeast recovery rates on three media

\begin{tabular}{lcccc}
\hline & \multicolumn{4}{c}{ Mean percentage recovery rate* } \\
\cline { 2 - 5 } Medium & C. tropicalis & C. albicans & C. glabrata & S. cerevisiae \\
\hline Sabouraud & 68.2 & 113.9 & 85.7 & 94.2 \\
Candida ID & 110.0 & 133.8 & 90.2 & 94.2 \\
\hline * Compared to blood agar. & & &
\end{tabular}




\section{Detection and presumptive identification of yeasts}

In all, 92 of the 240 clinical specimens yielded one or more yeast strains (79 pure cultures, 13 mixed cultures) on either Sabouraud-chloramphenicol or Candida ID media. The remaining 148 specimens were negative. A total of 105 yeast strains belonging to 9 species was isolated on at least one of the two media (Table 2). Table 2 shows that the overall detection rates of the two media were very similar. Sabouraud-chloramphenicol agar detected $86.7 \%(91 / 105)$ and Candida ID $85.7 \%$ $(90 / 105)$ of the total number of yeasts isolated. However, 11 mixtures of yeasts were detected on Candida ID, but only 5 on Sabouraud-chloramphenicol, a significant difference $(p<0.01)$. Table 3 shows the distribution of yeasts in the mixtures.

Of the 41 specimens that yielded $C$. albicans in pure culture on the Candida ID medium, 34 yielded growth at $24 \mathrm{~h}$. Blue pigmentation was found in $52.9 \%(18 / 34)$ at $24 \mathrm{~h}$ and $94.1 \%(32 / 34)$ at $48 \mathrm{~h}$. All blue colonies at $48 \mathrm{~h}$ were identified as C. albicans (specificity $100 \%$ ). Two strains of $C$. tropicalis were identified. In one case pink pigmentation appeared after incubation for $72 \mathrm{~h}$. The second isolate did not produce any pink pigmentation after incubation for 5 days, i.e., it was a false negative result. The strain of Pichia anomala produced pink pigmentation.

On the Candida ID medium, bacterial colonies could be easily distinguished from yeasts by their clear, glossy appearance.

\section{Discussion}

Analysis of recovery rates found Candida ID to be equivalent to Sabouraud-chloramphenicol agar in the detection of yeasts. This was reflected in the clinical samples, where detection rates differed by only $1 \%$. However, colonies of $S$. cerevisiae were smaller than on Sabouraud-chloramphenicol, which should be considered in laboratories attempting to detect $S$. cerevisiae. Candida ID was superior to Sabouraud-chloramphenicol

Table 2. Yeasts detected on Sabouraud-chloramphenicol (SC) and Candida ID medium

\begin{tabular}{lccc}
\hline & \multicolumn{3}{c}{ Number of yeasts detected on } \\
\cline { 2 - 4 } Yeast & SC & Candida ID & Any medium \\
\hline C. albicans & 49 & 48 & 54 \\
C. glabrata & 20 & 21 & 23 \\
S. cerevisiae & 14 & 9 & 15 \\
C. inconspicua & 3 & 3 & 3 \\
C. krusei & 3 & 3 & 3 \\
C. tropicalis & 0 & 2 & 2 \\
Pichia sp. & 0 & 2 & 2 \\
Pichia anomala & 1 & 1 & 1 \\
C. parapsilosis & 0 & 1 & 1 \\
Not identifiable & 1 & 0 & 1 \\
Total & 91 & 90 & 105 \\
\hline
\end{tabular}

Table 3. Mixtures of yeasts identified by Sabouraudchloramphenicol (SC) and Candida ID media

\begin{tabular}{lccc}
\hline & \multicolumn{2}{c}{ Number of mixtures detected on } \\
\cline { 2 - 4 } Organisms in mixture & SC & Candida ID & $\begin{array}{c}\text { Any } \\
\text { medium }\end{array}$ \\
\hline C. albicans/C. glabrata & 1 & 3 & 3 \\
C. albicans/C. tropicalis & 0 & 2 & 2 \\
C. albicans/S. cerevisiae & 1 & 1 & 2 \\
C. krusei/S. cerevisiae & 2 & 2 & 2 \\
C. albicans/C. parapsilosis & 0 & 1 & 1 \\
S. cerevisiae/Pichia sp. & 0 & 1 & 1 \\
Pichia anomala/Pichia sp. & 0 & 1 & 1 \\
S. cerevisiae/Not identifiable & 1 & 0 & 1 \\
Total & 5 & 11 & 13 \\
\hline
\end{tabular}

in the detection of mixtures of yeasts. The blue pigmentation of $C$. albicans allows other Candida species to be easily identified in mixed cultures. In simulated mixtures of up to three yeasts, there was good differentiation of blue, pink and white colonies after incubation for $48 \mathrm{~h}$.

The single previous evaluation of Candida ID used collection strains and strains from clinical samples but did not analyse simulated mixed cultures [8]. It showed equivalent overall detection rates to Sabouraud agar with superior detection of mixtures. Overall sensitivity for the detection and differentiation of C. albicans was $97.6 \%$ (after $72 \mathrm{~h}$ ). However, that study did not analyse rates of identification at 24 and $48 \mathrm{~h}$ and did not examine clinical specimens from a defined patient population. The results of the present study show rapid direct identification of C. albicans from clinical haematology surveillance samples, where prompt identification is important because of the high risk of invasive yeast infection. Rates of presumptive identification of C. albicans at 24 and $48 \mathrm{~h}(52.9 \%, 94.1 \%)$ are similar to previous studies by Rouselle et al. [4] (56\%, 90.9\%) and Freydière et al. [9] $(56.5 \%, 92.75 \%)$ with Albicans ID medium. The specificity of the blue pigmentation on Candida ID was $100 \%$ at $48 \mathrm{~h}$, also similar to previous study results of $98.6 \%$ [4] and 100\% [9]. Candida ID has been shown to have only $89.7 \%$ specificity for the detection and differentiation of C. albicans [8], but this was mainly due to the isolation of $C$. dubliniensis, which also produces blue pigmentation on the medium. Discrimination between these species is also difficult on other chromogenic media, but as their clinical manifestations and antifungal susceptibilities are very similar this should not affect patient management [10].

Although Candida ID has not been compared to other types of chromogenic media, a study has been performed comparing Albicans ID to CHROMagar and Candiselect (Sanofi Diagnostics Pasteur, France) [9]. Albicans ID had a better rate of direct identification of C. albicans at $24 \mathrm{~h}(56.5 \%$ versus $37.68 \%$ and $11.59 \%$ for Candiselect and CHROMagar, respectively), although at $72 \mathrm{~h}$ results were similar $(92.75 \%$, 
$91.3 \%$ and $88.57 \%$, respectively). Specificity of pigmentation for C. albicans was $100 \%$ for all three media at $72 \mathrm{~h}$. However, only CHROMagar Candida gave presumptive identification of C. tropicalis and C. krusei (at $48 \mathrm{~h}$ ). Although chromogenic media are more expensive than Sabouraud-chloramphenicol agar, the use of CHROMagar Candida has been found to be more cost-effective and less time-consuming than conventional methods (germ-tube test and API 20C AUX) for identification of yeasts after initial culture [11]. The latter study found the use of CHROMagar Candida as the primary isolation medium to be only marginally more expensive than Sabouraud-chloramphenicol agar when costs of further identification were taken into account. A further study has confirmed CHROMagar Candida as a more cost-effective yeast identification scheme (when combined with morphology on corn meal-Tween 80 agar) than API 20C AUX [12]. The current cost of pre-prepared Candida ID plates (£12.47 for 20 plates) compares favourably with CHROMagar Candida ( $£ 31.42$ for 20 plates). However, some of this difference may be offset by further identification costs for Candida ID, as CHROMagar Candida has a broader species range.

The present study examined the usefulness of the additional substrate producing pink colonies on Candida ID. Two isolates of $C$. tropicalis were found in clinical samples. However, one produced pink coloration only after incubation for $72 \mathrm{~h}$, and the other did not reveal any pink coloration even after 5 days. Product information from bioMérieux has shown that colonies of $C$. tropicalis and $C$. guilliermondii are usually pink after 48-72 h. However, it has been recognised that a few strains of these species remain white. It has also been acknowledged that some species (such as C. famata, C. humicola and Cryptococcus neoformans) may, in some cases, have pink coloration. In the present study, Candida ID offered little advantage over Albicans ID for the haematology population, because only two isolates of $C$. tropicalis were found. However, other haematology units may have a higher prevalence of $C$. tropicalis or other yeasts producing pink pigmentation, so that use of Candida ID would be of benefit.

This study has shown Candida ID to be equivalent to Sabouraud-chloramphenicol agar for the detection of yeasts and superior for detecting mixtures of yeasts.
This reflects previous experience with Candida ID, Albicans ID and CHROMagar media. It provides rapid and specific identification of $C$. albicans, therefore obviating the need for germ-tube testing. Candida ID may be suitable for use as a primary isolation medium because of its rapid identification of $C$. albicans, the commonest yeast isolated from clinical specimens, as well as its ability to identify mixed cultures of yeasts.

We thank bioMérieux for providing Candida ID plates and API 20C AUX identification kits. We are also grateful to the staff on Hogarth Ward, Nottingham City Hospital, for the collection of clinical specimens and to the laboratory technical staff for processing of specimens.

\section{References}

1. Jarvis WR. Epidemiology of nosocomial fungal infections, with emphasis on Candida species. Clin Infect Dis 1995; 20: $1526-1530$.

2. Wingard JR. Importance of Candida species other than $C$. albicans as pathogens in oncology patients. Clin Infect Dis 1995; 20: $115-125$.

3. Wingard JR, Merz WG, Rinaldi MG, Johnson TR, Karp JE, Saral R. Increase in Candida krusei infection among patients with bone marrow transplantation and neutropenia treated prophylactically with fluconazole. $N$ Engl J Med 1991; 325: 1274-1277.

4. Rousselle P, Freydiere A-M, Couillerot P-J, de Montclos H, Gille Y. Rapid identification of Candida albicans by using Albicans ID and Fluoroplate agar plates. J Clin Microbiol 1994; 32: 3034-3036.

5. De Champs C, Lebeau B, Ambroise-Thomas P, Grillot R. Evaluation of Albicans ID plates. J Clin Microbiol 1995; 33: 2227-2228.

5. Odds FC, Bernaerts R. CHROMagar Candida, a new differential isolation medium for presumptive identification of clinically important Candida species. J Clin Microbiol 1994; 32: $1923-1929$.

7. Willinger B, Manafi M. Evaluation of CHROMagar Candida for rapid screening of clinical specimens for Candida species. Mycoses 1999; 42: 61-65.

8. Fricker-Hidalgo H, Orenga S, Lebeau B et al. Evaluation of Candida ID, a new chromogenic medium for fungal isolation and preliminary identification of some yeast species. J Clin Microbiol 2001; 39: 1647-1649.

9. Freydière AM, Buchaille L, Gille Y. Comparison of three commercial media for direct identification and discrimination of Candida species in clinical specimens. Eur J Clin Microbiol Infect Dis 1997; 16: 464-467.

10. Sebti A, Kiehn TE, Perlin D et al. Candida dubliniensis at a cancer centre. Clin Infect Dis 2001; 32: 1034-1038.

11. Ainscough S, Kibbler CC. An evaluation of the costeffectiveness of using CHROMagar for yeast identification in a routine microbiology laboratory. J Med Microbiol 1998; 47: $623-628$.

12. Koehler AP, Chu K-C, Houang ETS, Cheng AFB. Simple, reliable, and cost-effective yeast identification scheme for the clinical laboratory. J Clin Microbiol 1999; 37: 422-426. 